Dermatologische Zeitschrift. 1916;23:I-V

\title{
Contents, Vol. 23, 1916
}

\section{Dermatologische Zeitschrift}

Begründet von O. LASSAR

Unter Mitwirkung von

A. Alexander-Berlin; J. Almkvist-Stockholm; Ed. Arning·Hamburg; F. Bering-Essen; S.

Bettniann - Heidelberg; B. Bloeh - Zurich; C. Bruhns - Berlin; A. Buschke-Berlin; C.

Cronquist-Malmö; J. Darier-Paris; K. Dohi-Tokio; S, Ehrmann-Wien; J. Fabry-Dortmund; W.

Frieboes-Rostock; W. Gennerich-Kiel; C. Grođven-Halle; L. Hauck-Erlangen; J. Heller-Berlin;

W. Heuck-München; H. Ïiübner-Elberfeld; A. Jesionek-Gießen; F. Juliusberg-Posen; F. Kyrle-

Wien; A. Lanz-Moskau; P. Linser-Tübingen; R. Matzenauer-Graz; G. Nobl-Wien; A. Pasini-

Mailand; F. Pinkus-Berlin; R. Polland-Graz; C. Raseh-Kopenhagen; E. Riecke-Leipzig; G. Rosí-

Freiburg; A. Ruete-Marburg ; J. Schaeffer-Breslau; G. Seherber-Wien; H. E.Schmidt-Berlin; W.

Scholtz-Königsberg i. Pr.; B.Spiethoff-Jena; C. Stern-Düsseldorf; E. Tomasczewski-Berlin; L. ТӦГӦk-ßuda-pest; H. VÖrner-Leipzig; J. Werther-Dresden; Ch. J. White-Boston; J. ZeislerChicago; K. Zieler-Würzburg; F. Zinsser-Köln a. Rh.

und in Gemeinschaft mit

G. Ärndt A. Biaschko S. Ehrmann K. Herxheimer V. Klingmüller

Straßburg Berlin Wien Frankfurt a. M. Kiel

Herausgegeben von

E. Hoffmann

Bonn

Bd. XXIII.

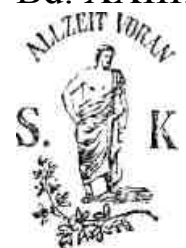

berlin í9í6

vérlag von s. karger

KARLSTRASSE 15.

]Nit zahlreichen Abbildungén im Text und $1 \delta$ Tafeln.

Alle Recht $\theta$ vorbehaiten.

Gedruckt bei Imberg \& Lefson G. m. b. H. in Berlin SW. 48.

Inhalts-Verzeichnis.

Original- Arbeiten. Selte

A lexander, Arthur und MaxZenger, Über Atrophia

cutis maculosa luetica. (Hierzu Tafel VII) 1

Almkvist, Johan, Über die primären ITrsprungsstellen

und die sekundäre Ausbreitung der merkuriellen ulzerösen

Stomatitis und über die Entstehung der Salivation bei 
Quecksilberbehandlung. (Hierzu Tafel I-VI) ... 14, 79 Altmann, K. , Zur Abortivbehandlung der Syphilis . . . 257 Bender, Julie, Über die Degenerationsformen der Gono-

kokken und Einlagerungen in den Epithelien des gonorrho-

ischen Eiters 577

Björling,E., Annuläre Formen von Dermatitis chronica

atrophicans 425

Blumen th a 1, Franz und Dr. T sakalotis, Zur

quantitative!! Bestimmung der Syphilisstoffe

C o 11 m a n n, Carl, Die Färbemethoden nach Much und

Ziehl zum Nachweis von Tuberkelbazillen im Gewebe . .321 Cronquist, C a r 1, Seltene

Lokalisation von Primär-

affekten

44

- , Über die Bedeutung der Skleradenitiden bei der Beur-

teilung der Heilung der Syphilis 288

F ö n s s , A a g e , L., Maniíeste unbehandelte Syphilis mit

negativer Wassermannscher Reaktion 212

$\mathrm{G}$ e $\mathrm{n} n$ e r i c h, W.. Über eine im Schützengrabendienst er-

worbene progressive, diffuse Hautatrophie an beiden

Beinen. (Hierzu Tafel XVI-XVII) . 673

Gerlach, K. W., Weißwerden der Haare und Haaraus-

fall nach psychischem Trauma oder Vitiligo mit sebor-

rhoischer Alopecie i 545

G j e s s i n g, E., Telangiectasia hereditaria hämorrhagica

(Osier) 193

Gold b e r g e r . E d u a r d, Zur Frage der Salvarsan-

behandl·ung bei weichen Schankergeschwüren .... 661 Gutmann, C, Zur Frage der

Weiterbehandlung mit

Salvarsan nach Auftreten eines Salvarsanxanthems . 338

- IV -

Seite

Haslund, P o u 1 , Granulosis rubra nasi. Scheinbar an-

dauernde Heilung mit Kohlensäureschnee 135

,$-(\dagger)$, Über das Vorkommen von Lupus erythematosus

auf dem Prolabium der Lippen und der Schleimhaut

des Mundes 705

Haxthausen, H., Epithelproliferationen, hervorgerufen

durch Einwirkung von Anilin auf die Haut 595

Heim, Gustav, Seltenheit des Lupus und der Psoriasis

in heißen Ländern 357

Hell, P., Soorerkrankung der hinteren Harnröhre (Uretliri-

lis posterior mycotica) mit Incontinentia urinae 641

Heller, Julius. EinigeklinischeBeobachtimgenangeschlechts-

kranken Soldaten 65

, Zur Kasuistik seltener Hagelerkrankungen. XII. Ony-

chogryphosis als Naehkrankheit des Herpes zoster .... 726

Herxheimer, Karl, Über die epidermidale Basalmem- 
bran. (Hierzu Tafel VIII) 129

Lindenheïm, H., Über das erste Stadium der Mycosis fungoides. (Hierzii Tafel IX-X) 459

M ü 11 e r, Rudolf, Über Sklerosebildung bei salvarsanbehandelten Syphilitikern. (Reininfeetio ? Sclerosis ex residuo ?) 395

Neißen, A., TJiskussionsbemerkungen zu Werthers Arbeit , Über einen Fall von Pityriasis lichenoides (besser polymorpha) chronica, welcher die drei Typen der Parapsoriasis vereint." 46 Perut z, Alfred, Zur pharmakologischen Behandlung des Erysipels 449 Polland, R., Zur Pathogenese der Epidermolysis bullosa hereditaria 385

-- -, Fibromatosis subungualis. (Hierzu Tafel XI) .... 542 Rosenthal, O., Über einige wichtige Fragen bei der Be handlung geschlechtskranker Soldaten, besonders über die Bedeutung der Wassermannschen Reaktion 74

Rost, G. A., Liquoruntersuchungen bei Syphilis . . 147, 236

$\mathrm{R} \mathrm{u}$ e t e , A. E., Beiträge zur Frage der Tuberkulide und des Lupus erythematodes. (Hierzu Tafel XII-XV) . 513, 602 Schlasberg, H. J., Über intravenöse Arthigoninjektionen zu diagnostischem Zweck Ill Stümpke, Gustav, Über multiple Talgdrüsenhypertrophien (Adenoma sebaceum) 296

V-

Seite

Werther, Johannes, Erwiderungzu den obigen Diskussionsbemerkungen Neißers 47

$\mathrm{Z}$ i e $\mathrm{m}$ a $\mathrm{n}$ n, L., Über Impetigo herpetiformis

Z i r n , C, Die Behandlung der Krampfadern mit intravenösen

Sublimatinjektionen . 650

Geseliseîiaftsberichte.

Berliner Dermatologische Geseilschaft. Sitzung vom 12. Oktober1915 163

Bericht über den III. Kongreß des Vereins Nordischer Dermatologen in Christiania. Erstattet von Dr. B. Pantoppidan in Kopenhagen , ,.551

Periodische Literatur. 48, 122, 182, 249, 305, 366, 431, 494, 557, 629, 688, 735

Buchanzeigen 63, 190, 317, 448, 511, 638

Tagesnachriehten und Personalien 192, 320, 384, 512, 576702

Nekrologe.
A. Wolff
P. Hasluncl 
Eduard Lang .... .

575

Sachregister

750

Namenregister

758 